Advances in Applied Ceramics, Volume 109, Issue 3, 2010, Pages 156-161

Bioceramics

Editorial Manager(tm) for Advances in Applied Ceramics: Structural, Functional and Manuscript Draft

Manuscript Number:

Title: Impedance measurements for determination of the elastic and piezoelectric coefficients of films

Article Type: Invited Response

Keywords: Resonance; Characterization; Piezoelectrics; Films; Iterative Method; Cantilevers; PZT

Corresponding Author: Prof. Dr. Lorena Pardo, Ph.D.

Corresponding Author's Institution: Into. Ciencia de Materiales de Madrid

First Author: Lorena Pardo, Ph.D.

Order of Authors: Lorena Pardo, Ph.D.; Ricardo Jiménez, Ph.D; Alvaro García, B.Sc.; Klaus Brebøl, Ph.D; Glen Leighton, Ph.D.; Zahorong Huang, Ph.D.

Abstract: Most of those techniques used for the measurement of elastic coefficients for bulk piezoelectric ceramics are not applicable to films deposited on thick substrates because the measured properties, such as the resonant frequency, are usually dominated by the presence of the thick substrate. This work presents a preliminary study for the application of Alemany et al. automatic iterative method to the determination, from complex impedance measurements, of the film properties using a conventional self-supported cantilever design used in MEMS applications and fabricated from a PZT thick film on Si-based substrate. 


\title{
Impedance measurements for determination of the elastic and piezoelectric coefficients of films
}

\author{
L. Pardo ${ }^{1}$, R. Jiménez ${ }^{1}$, A. García ${ }^{1}$, K. Brebøl ${ }^{2}$, G. Leighton ${ }^{3}$ and Z. Huang ${ }^{3}$ \\ ${ }^{1}$ Instituto de Ciencia de Materiales de Madrid (ICMM-CSIC). Cantoblanco. \\ 28049- Madrid (Spain) \\ ${ }^{2}$ Limiel ApS. Langebæk. (Denmark) \\ ${ }^{3}$ Department of Materials, School of Applied Sciences, Cranfield University, \\ Bedfordshire, MK43 0AL (UK)
}

\begin{abstract}
Most of those techniques used for the measurement of elastic coefficients for bulk piezoelectric ceramics are not applicable to films deposited on thick substrates because the measured properties, such as the resonant frequency, are usually dominated by the presence of the thick substrate. This work presents a preliminary study for the application of Alemany et al. automatic iterative method to the determination, from complex impedance measurements, of the film properties using a conventional self-supported cantilever design used in MEMS applications and fabricated from a PZT thick film on Si-based substrate.

\section{$\underline{\text { 1. Introduction }}$}

The resonance method for bulk piezoceramic characterization in the linear range is a commonly used technique developed long time ago and for which numerous Standars has been issued ${ }^{1,2}$. This method allows to determine a number of material coefficients from the measurement of the frequency dependence of the complex impedance at the electromechanical resonance modes of ferro-piezoelectric ceramics with given geometries. Iterative ${ }^{3,4}$ and fitting methods of analysis of the impedance spectra have been developed to provide and alternative to the limitations of the Standard calculation methods concerning the characterization of high loss and low sensitivity ferro-piezoelectric ceramic materials.

In the automatic iterative method developed at ICMM-CSIC the material data is determined by solving a set of non-linear equations that results when experimental impedance data at a number of frequencies are introduced into the appropriate analytical expression of the wave equation for a given electromechanical resonance mode. This set of equations is established for as many frequencies, which are automatically selected by the program, as unknown coefficients. Solution is carried out by an iterative numerical method ${ }^{3,4}$.
\end{abstract}


Such analytical expression are valid for sample geometries with given aspect ratios, which allows exciting uncopled modes. Regular sample geometries are recommended for the use of this characterization method and extreme care shall be taken to determine accurately the dimensions and density of the samples, required for the accurate determination of the complex material parameters.

It is worth noting that four resonance modes, that can be measured using three sample shapes (thickness-poled thin disk, thickness-poled shear plate and long rod or bar), are enough to get the full set of independent parameters (2 dielectric $\left(\varepsilon_{11}^{\mathrm{S}}\right.$ and $\left.\varepsilon_{33} \mathrm{~S}_{33}\right), 3$ piezoelectric $\left(\mathrm{d}_{33} \mathrm{~d}_{31}\right.$ and $\left.\mathrm{d}_{15}\right)$ and 5 elastic $\left.\left(\mathrm{s}^{\mathrm{E}}{ }_{11}, \mathrm{~s}^{\mathrm{E}}{ }_{12}, \mathrm{~s}^{\mathrm{E}}{ }_{33}, \mathrm{~s}^{\mathrm{E}}{ }_{13}, \mathrm{~s}^{\mathrm{E}}{ }_{44}\right)\right)$ that characterizes a ferro-piezoelectric poled ceramic material ( $6 \mathrm{~mm}$ symmetry). Iterative methods were developed for all these resonance modes, which allowed a consistent and accurate matrix characterization of this type of materials ${ }^{5}$.

Since most of the ferro-piezoceramic thin and thick films also have such $6 \mathrm{~mm}$ symmetry, in principle this method can be extended also to the film characterization. However, in fact they are not applicable to thin films deposited on thick substrates because the measured properties, such as the resonant frequency, are usually dominated by the presence of the thick substrate and their analytical treatment is not straightforward ${ }^{6}$. This work presents a preliminary study for the application of Alemany et al. automatic iterative method ${ }^{3,4}$ to the determination, from complex impedance measurements, of thick and thin film properties, using a conventional selfsupported bending cantilever design used in MEMS applications and fabricated from a PZT film on Si substrate ${ }^{6}$.

\section{$\underline{\text { 2. Experimental }}$}

\subsection{Self-supporting Piezoelectric Cantilevers from Thick Films}

The substrate for the fabrication of the freestanding $\mathrm{Pb}_{1.2}\left(\mathrm{Zr}_{0.52} \mathrm{Ti}_{0.48}\right) \mathrm{O}_{3}$ cantilevers was a $350 \mu \mathrm{m}$ thick double side polished $<100>$ silicon (Si) wafer, with surface oxide $\left(\mathrm{SiO}_{2}\right)$ of $200 \mathrm{~nm}$ thickness. The initial processing step was to prepare the wafer for the growth of the piezoelectric layer, this involved the prevention of lead diffusion into the Si wafer through the bottom electrode, which can form a liquid phase lead silicate at the annealing temperatures used for the piezoelectric $\left(\sim 720^{\circ} \mathrm{C}\right)$, with the use 
of a $60 \mathrm{~nm}$ thick zirconium oxide layer $\left(\mathrm{ZrO}_{2}\right)$. The $\mathrm{ZrO}_{2}$ was deposited with a solgel method where a solution is prepared from $47 \mathrm{ml}$ of ethanol anhydrite at $99.9 \%$, $3 \mathrm{ml}$ acetic acid and $4.344 \mathrm{~g}$ of zirconium propoxide, all of which are measured under an $\mathrm{N}_{2}$ ambient atmosphere. The chemicals were then combined and stirred for 60 minutes. The three layers of $\mathrm{ZrO}_{2}$ each of which were dried at $200^{\circ} \mathrm{C}$ and pyrolised at $350^{\circ} \mathrm{C}$, before subsequent crystallisation of the complete $\mathrm{ZrO}_{2}$ layer was performed in a rapid thermal annealer (RTA) at $800^{\circ} \mathrm{C}$. The wafer was platinised using sputtering in a Nordiko RF/DC sputtering machine, $8 \mathrm{~nm}$ of RF sputtered Ti was used as an adhesion layer for the $100 \mathrm{~nm}$ thick DC sputtered Pt to act as a the bottom electrode. The thick film lead zirconate titanate (PZT) is composed of a composite slurry containing Ferroperm PZ26 PZT powder mixed with PZT sol to create the bulk of the PZT layer, which due to its high porosity is infiltrated with a PZT sol diluted with 2Methoxy-Ethanol with a 1:1 ratio to increase densification to $7,07 \mathrm{~g} / \mathrm{cm}^{3}$. The process to deposit a complete layer of PZT uses two depositions of the PZT slurry, with 4 four sol infiltration steps giving a 'layer' thickness of $3.4 \mu \mathrm{m}$. Each component of the 'layer' of the PZT slurry and sol infiltration is deposited onto the substrate in a cyclic process which includes drying at $200^{\circ} \mathrm{C}$ and Pyrolosis $450^{\circ} \mathrm{C}$ of the PZT film to provide the required total thickness in our case around $10 \mu \mathrm{m}$, prior to sintering in a box furnace at $720^{\circ} \mathrm{C}$. A lift-off lithography process incorporating LOR2A and S1818 resists was used to deposit a patterned top electrode with the same parameters as the bottom electrode. Following the deposition of the top electrode and subsequent lift off, the wafer was RF sputter coated with a blanket layer of gold $200 \mathrm{~nm}$ thick, a 2.5 $\mu \mathrm{m}$ thick resist mask was patterned upon the gold layer using the image reversal resist AZ5214E to provide a mould. $1 \mu \mathrm{m}$ of nickel was then electroplated into the resist mould which was then stripped giving a hard metal mask for the deep reactive ion etching (DRIE) of the PZT film ${ }^{7}$ After the etch of the PZT, the hard mask was removed by wet etching the nickel in ferric chloride, and removing the gold in potassium iodide and iodine. The wafer was then patterned using AZ4562 resist to RIE etch the bottom electrode and the surface oxide with $\mathrm{Ar}$ and $\mathrm{CHF}_{3}, \mathrm{O}_{2}$ gasses respectively. After this the wafer was patterned on the back face using the same image reversal technique as the front to allow the sputter deposition and subsequent lift off of a $100 \mathrm{~nm}$ thick aluminium layer which acts as the hard mask for DRIE through the Si wafer to release the dies. The final step is the $\mathrm{O}_{2}$ ashing of the 
protective resist on the front of the wafer and chemical cleaning using acetone and isopropanol alcohol. The devices obtained for this study are shown in Figure 1.

\subsection{Impedance measurements}

A special measurement cell was fabricated for the purpose of accurate connection to the conducting paths of the devices and testing under reduced pressure conditions (below 1bar) the evolution of the resonance spectra (Figure 2). Measurements were carried out using an HP4192A LF impedance analyser.

The first fact to take into account for this measurements is that the measuring voltage must be fixed so as to stablish a linear range. Figure 3 shows the evolution of the measured resistance and impedance for the L26 device as a function of the voltage applied to the sample. Asymmetric peaks are found for $50 \mathrm{mV}$ or higher. Such an effect is well know as to be due to non-linear behaviour and has also been observed in ceramics under $50 \mathrm{~V}$ of excitation ${ }^{8}$.

The second fact that must be considered is the ocurrence of coupling between resonance modes, since the method under study is only valid for single modes. Figure 4 shows the resistance, $\mathrm{R}$, and conductance, $\mathrm{G}$, at the resonances of devices L10 and L26. Whereas the spectrum for L10 shows three peaks, corresponding to three modes of vibration, the L26 device shows an uncoupled unique mode of resonance.

\subsection{Analysis of modes of resonance and principles for the calculation}

The identification of the mode of movement of the resonance to be used for the parameters calculation is a crucial issue for the validity of the method.To determine the mode of motion FEA simulation of the cantilevers was made using ATILA software (9) and a three-dimentional harmonic analysis. Due to the symmetry of the system, only one half of the device was modelled to reduce the calculation time. Bending motion of L28 was found at $775 \mathrm{~Hz}$, whereas a pure length extensional mode was found at $292 \mathrm{kHz}$ and a coupled torsional movement and wavy length extensional mode was found at $325 \mathrm{kHz}$. Similar behaviour was found for L10, at higher frequencies since the cantilever length is lower (Figure 4(a)). Such modes of resonance are very close in frequency and coupling can be expected, thus are not optimum for the calculation of the film parameters by the method here studied. 
Bending motion of L26 was found at $2260 \mathrm{~Hz}$, whereas at $488 \mathrm{kHz}$ a pure length extensional mode is found. Thus the resonace $R$ and $G$ peaks shown in Figure 4(b) are identified as the lenght extensional mode of the cantilever L26.

Alemany et al. developed an automatic iterative method for piezoceramic characterization that provides the values of $\varepsilon^{\mathrm{T}}{ }_{33}, \mathrm{~s}^{\mathrm{E}}{ }_{11}$ and $\mathrm{d}_{31}$, as well as the electromechanical coupling factor $\mathrm{k}_{31}$, from the impedance measurements at the length extensional mode of a thickness poled long bar $^{3}$, which can be used for the purpose of characterization from the mode shown in Figure 4(b). For the thickness poled cantilever ( $\lambda / 4$ wave resonator) we have a lenth extensional mode of motion with a node at one edge, where the cantilever is fixed to the substrate, and maximum amplitude at the other edge. The distribution of strain in the cantilever was obtained by FEA and it is shown in Figure 5(a). A thickness poled bar of double lenght and double capacitance of the cantilever ones will have a node at the center and maximum amplitudes at both ends ( $\lambda / 2$ wave resonator), as FEA results in Figure 5(b) shows. Both resonators share contour conditions and the analytical expression and resonance frequency is the same for both. Making appropriated geometric corrections it is possible to calculate the material parameters of the cantilever for the mentioned mode using the software of the Alemany method for the length extensional resonance of thickness poled bar.

\section{Results and discussion}

The above mentioned calculation was made for the L26 device from the measurements at the verge of the linear range, $10 \mathrm{mV}$ input signal, determined after measurements in the range of 5 to $100 \mathrm{mV}$ (Figure 3). The results of the calculated parameters and the coupling factor, $\mathrm{k}_{31}$, are shown in Table I. The regresion factor, $\mathrm{R}^{2}$, of the reconstructed spectra using the calculated parameters to the experimental one is high, indicating that the calculus is accurate. However, the high value of $\varepsilon_{33}{ }^{\mathrm{T}}$ obtained indicates that admittance data are overstimated. In fact, the capacitance measured below the conducting paths, which are needed to conect the cantilever to the measuring equipment, has a value of the same order of the capacitance of the cantilever. Therefore, the measured admittance needs a correction arising from this stray capacitance in parallel with the cantilever. This stray capacitance was calculated 
from the total value measured and the ratio between the area of the conducting paths to that of the total electroded area of the device. After this correction is made, considering negligible the resitance of the conducting paths, in addition to find a lower value of $\varepsilon_{33}{ }^{\mathrm{T}}$ at resonance, we found that the coupling factor increases. The elastic coefficient remains unchanged after the data correction

\section{$\underline{\text { Conclusions }}$}

The feasibility of the use of the resonance method for the calculation of the dielectric, elastic and piezoelectric complex parameters, as well as coupling coefficients of selfsupporting thin or thick films has been shown. Alemany et al. iterative method for the length extensional resonance mode of thin bars, thickness poled and excited, provide a way of calculation of complex film parameters $\varepsilon^{\mathrm{T}}{ }_{33}, \mathrm{~s}_{11}^{\mathrm{E}}$ and $\mathrm{d}_{31}$, as well as the electromechanical coupling factor $\mathrm{k}_{31}$, from the resonance of a self-supported thick film cantilevers.

Special care must be taken in the complex admittance measurements concerning the linearity regime of the measurement and the residual capacitance of the connecting pads, which influences both the dielectric and piezoelectric properties determination. Work is under progress to asses the best poling conditions, loading effects of the top electrodes and effect of reduced pressure atmosphere for the accurate determination of the material parameters of thin and thick films.

\section{Acknowledgements}

Thanks are given to Multifunctional and Integrated Piezoelectric Devices (MIND) Network of Excellence (FP6 515757-2 CE contract) for funding this work. 


\section{References}

(1) "IEEE Standard on piezoelectricity". ANSI/IEEE Std. 176-1987.

(2) "Piezoelectric properties of ceramic materials and components. Part 2: methods of measurement - Low power". European Standard CENELEC, EN 50324-2.

(3) C. Alemany, L. Pardo, B. Jiménez, F. Carmona, J. Mendiola and A.M. González. "Automatic iterative evaluation of complex material constants in piezoelectric ceramics". J. Phys. D: Appl. Phys. 27, 148 (1994).

(4) C. Alemany, A.M. González, L. Pardo, B. Jiménez, F. Carmona and J. Mendiola. "Automatic determination of complex constants of piezoelectric lossy materials in the radial mode". J. Phys. D: Appl.Phys. 28, 945-956 (1995).

(5) L. Pardo, M. Algueró and K. Brebøl ."A Non-Standard Shear Sample for the Matrix Characterization of Piezoceramics and its Validation Study by Finite Element analysis". J. Phys. D: Appl. Phys. 40 , 2162-2169 (2007).

(6) M. Lucas, T. Olding, M. Sayer, R. Tasker and S. Sherrit. "Thickness mode material constant of a supported piezoelectric film". J. Appl. Phys. 85(5), 2835 (1999).

(7) Z. Huang, G. Leighton, R. Wright, F. Duval, H.C. Chung, P. Kirby and R.W. Whatmore."Determination of piezoelectric coefficients and elastic constant of thin films by laser scanning vibrometry techniques". Sensors and Actuators A 135, 660665 (2007).

(7) Wang, S. Li, W. Wakabayashi, K and Esashi, M. "Deep reactive ion etching of lead zirconate titanate using sulphur hexafluoride gas". J. Am. Ceram. Soc. 82 (5) 1339-41 (1999).

(8) J. F. Blackburn and M.G. Cain."Non-linear piezoelectric resonance analysis using burst mode: a rigurous solution”. Journal of Physics D: Appl. Phys. 40(19, 227 (2007).

(9) 1997 ATILA User's Manual Institut Superieur d'Electronique du Nord, Acoustics Laboratory. 


\section{List of Figure captions}

Figure 1. Devices obtained for the study. Film thickness is $10 \mu \mathrm{m}$, width $250 \mu$ and length 2500, 2000 and $1500 \mu \mathrm{m}$ (from left to right).

Figure 2. Sample holder and example of experience with reduced atmosphere.

Figure 3. Resistance, R, and conductance, G, measured at the resonance of device L26 as a function of the measuring voltage.

Figure 4. Resistance and Conductance measured at the resonances of devices (a) L10 and (b) L26.

Figure 5. Strain distribution at two equivalent resonance modes: (a) the $\lambda / 4$ length extensional mode of a thickness poled cantilever of thickness $t$, width $\mathrm{w}$ and length 1 and (b) the $\lambda / 2$ length extensional mode of a thickness poled bar of thickness $t$, width $\mathrm{w}$ and length $2 \mathrm{xl}$. 
Table I. Resonance characteristics and thick film parameters of L26 device .

\begin{tabular}{|c|c|c|}
\hline $\begin{array}{l}\text { PARAMETERS RELATED TO THE } \\
\text { FUNDAMENTAL LENGTH } \\
\text { EXTENSIONAL RESONANCE MODE } \\
\text { OF CANTILEVERS, THICKNESS } \\
\text { POLED AND EXCITED }\end{array}$ & $\begin{array}{c}\text { Raw measured data } \\
\text { L26 sample measured } \\
\text { at } 10 \mathrm{mV}\end{array}$ & $\begin{array}{c}\text { Corrected data } \\
\text { L26 sample measured } \\
\text { at } 10 \mathrm{mV}\end{array}$ \\
\hline $\mathrm{F}_{\mathrm{S}}(\mathrm{kHz})$ & 493.82 & 493.82 \\
\hline $\mathrm{F}_{\mathrm{p}}(\mathrm{kHz})$ & 494.03 & 495.08 \\
\hline Auxiliary Frequencies & $\begin{array}{l}\mathrm{F} 1=486.26 \mathrm{kHz} \\
\mathrm{F} 2=501.74 \mathrm{kHz}\end{array}$ & $\begin{array}{l}\mathrm{F} 1=485.52 \mathrm{kHz} \\
\mathrm{F} 2=499.07 \mathrm{kHz}\end{array}$ \\
\hline Number of iteracions & 491 & 33 \\
\hline $\mathrm{R}^{2}$ & 0.999937 & 0.999915 \\
\hline$\left|K_{31}\right|$ & $3.13 \div$ & $7.71 \div$ \\
\hline $\mathrm{N}_{31}(\mathrm{kHz} \cdot \mathrm{mm})$ & 1481 & 1482 \\
\hline$s_{11}^{E}\left(10^{-12} \mathrm{~m}^{2} \mathrm{~N}^{-1}\right)^{*}$ & $16.10-0.05 i$ & $16.10-0.05 i$ \\
\hline$d_{31} \quad\left(10^{-12} \quad C \cdot N^{-1}\right)$ & $-18,4+0.7 i$ & $-13.0+0.5 i$ \\
\hline$\varepsilon_{33}{ }^{\mathrm{T}}$ & $2415-58 i$ & $200-1.8 i$ \\
\hline
\end{tabular}

50

51 

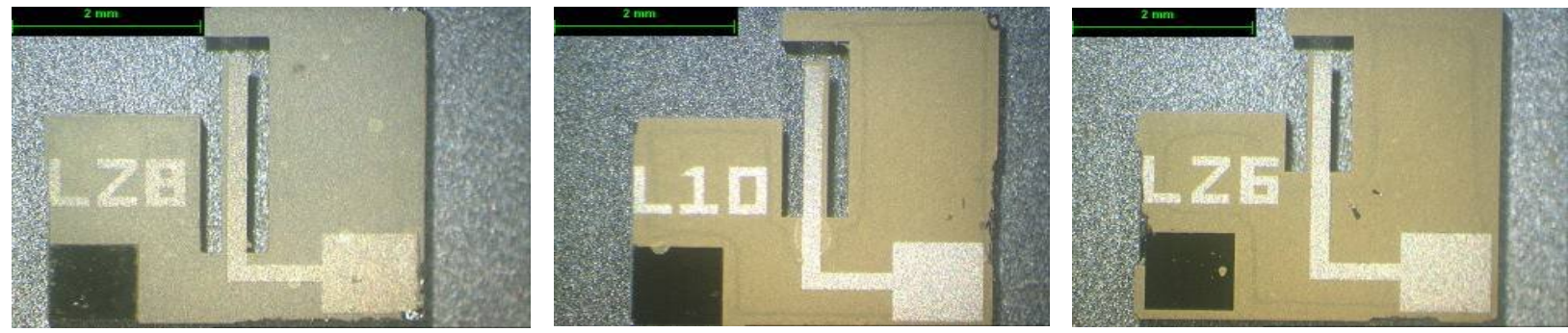

Figure 1. Devices obtained for the study. Film thickness is $10 \mu \mathrm{m}$, width $250 \mu$ and length 2500, 2000 and $1500 \mu \mathrm{m}$ (from left to right). 

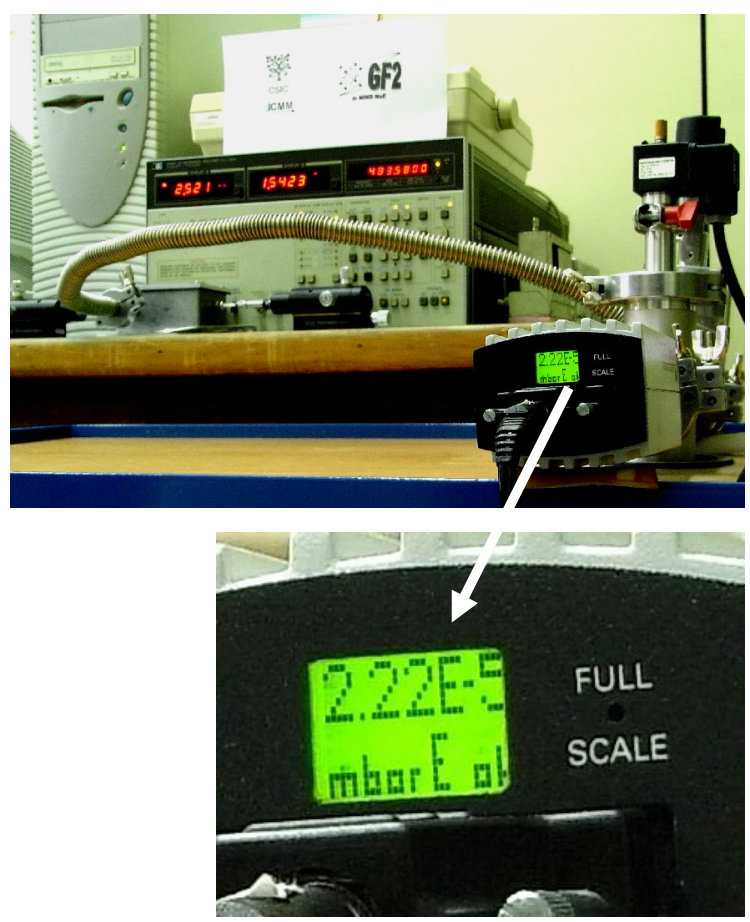

Figure 2. Sample holder and example of experience with reduced atmosphere. 

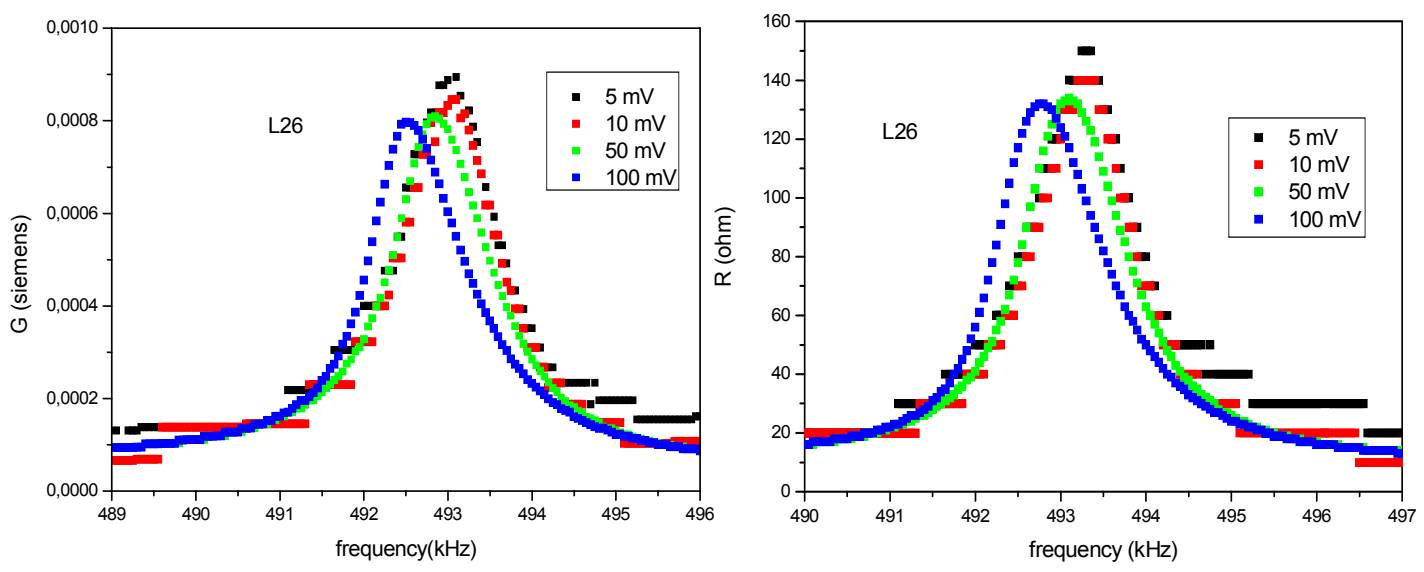

Figure 3. Resistance, R, and conductance, G, measured at the resonance of device L26 as a function of the measuring voltage. 
Figure 4. Resistance and Conductance measured at the resonances of devices (a) L10 and (b) L26.
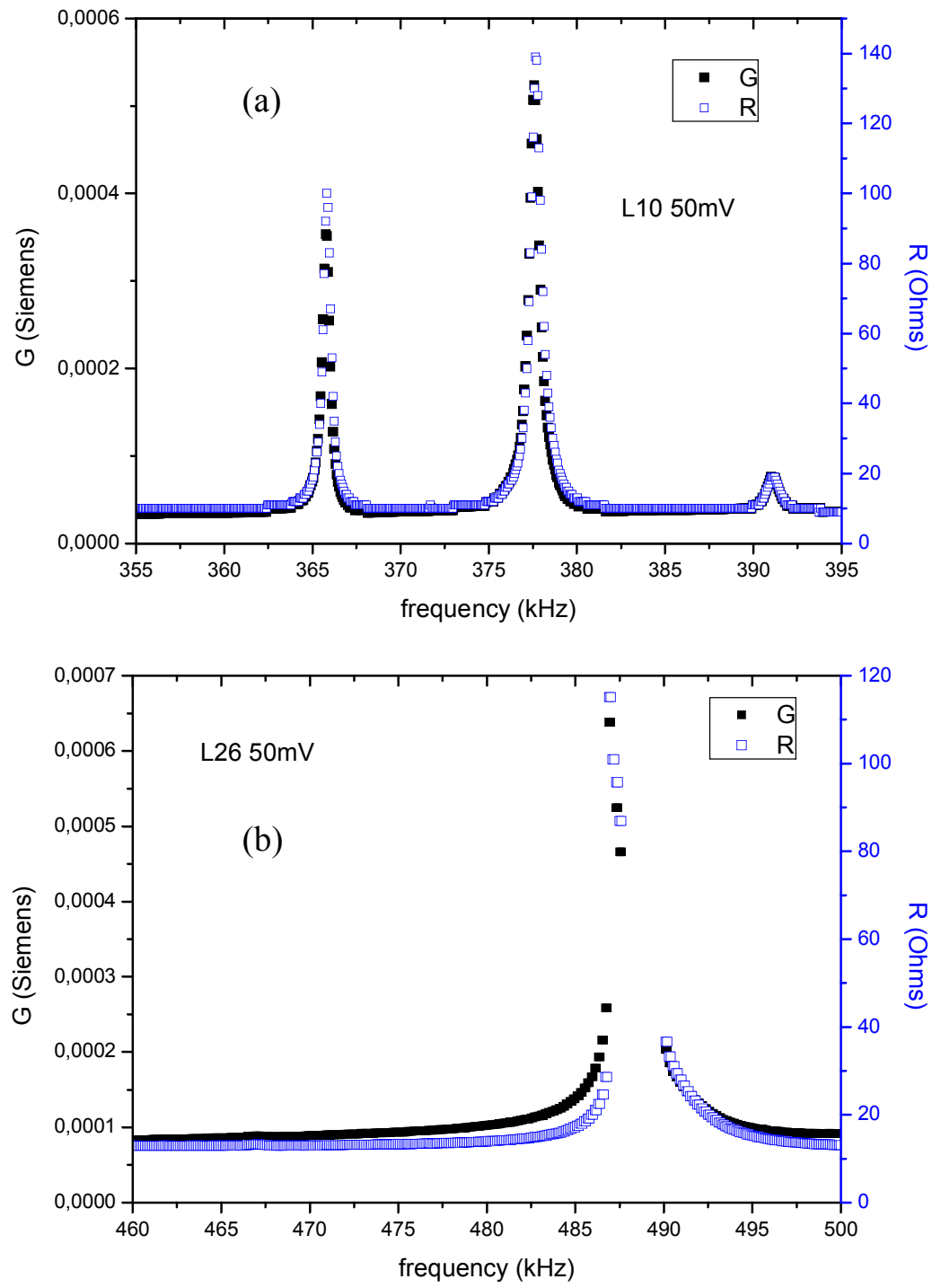


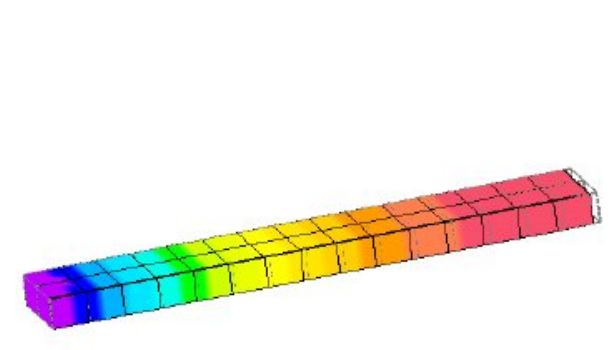

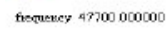
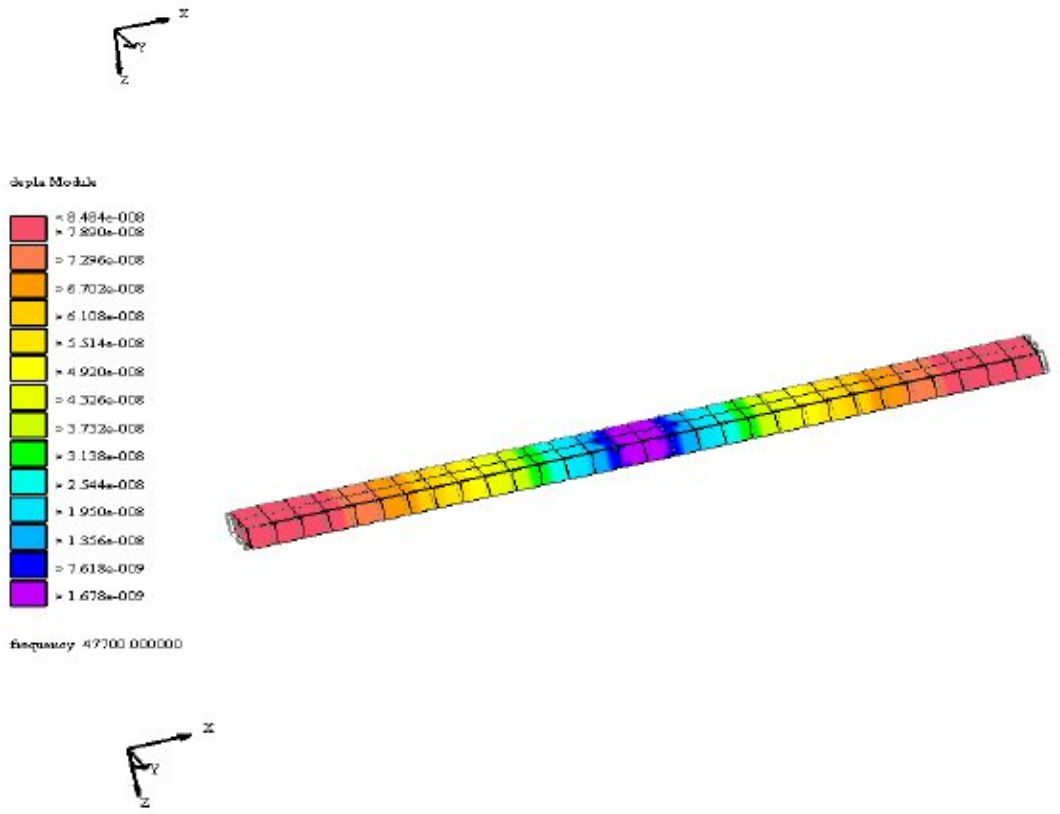

Figure 5. Strain distribution at two equivalent resonance modes: (a) the $\lambda / 4$ length extensional mode of a thickness poled cantilever of thickness $t$, width $\mathrm{w}$ and length 1 and (b) the $\lambda / 2$ length extensional mode of a thickness poled bar of thickness $t$, width w and length $2 \times 1$. 